Mönning

Betriebsfortführung in der Insolvenz 



\title{
Betriebsfortführung in der Insolvenz
}

\author{
2., neu bearbeitete Auflage \\ 2014 \\ herausgegeben von \\ Professor Dr. Rolf-Dieter Mönning
}

bearbeitet von

Daniel Bauch, Prof. Dr. Christian Berger, Friedrich Birnbreier, Dr. Jochen Blöse, Dr. Mark Boddenberg,

Charalambos Bograkos-Tzannetakos, Eric Coordes, Detlev Cornelius, Dr. Friedrich L. Cranshaw, Artur Deichmann, Prof. Dr. Martin Dreschers, Udo Feser, Robert Fliegner, Dr. Michael Flitsch, Dr. Michael C. Frege, Ingo Gerdes, Erwin Gerster, Dr. Marc Alexander Göb, Marion Gutheil, Ottmar Hermann, Dr. Frank Kebekus, Dr. Christoph Keller,

Prof. Ulrich Keller, Dr. Oliver Klöck, Alexander Langenmayer,

Dr. Ellen Meyer-Sommer, Michael Mönig, Prof. Dr. Rolf-Dieter Mönning, Dr. Matthias Nicht, Michael Pluta, Prof. Dr. Hanns Prütting, Knut Rebholz, Hans-Peter Runkel, Cornelia Schäfer, Prof. Dr. Jens M. Schmittmann,

Henning Schorisch, Jörg Spies, Prof. Dr. Wilhelm Uhlenbruck,

Dr. Sven-Holger Undritz, Holger Voskuhl, Dr. Stefan Weniger,

Dr. Carsten M. Wirth, Dr. Hermann Peter Wohlleben,

Wolfgang Zenker, Dr. Franc Zimmermann

\section{RWS}

RWS Verlag Kommunikationsforum GmbH · Köln 
Die Deutsche Nationalbibliothek verzeichnet diese Publikation in der Deutschen Nationalbibliografie; detaillierte bibliografische Daten sind im Internet über http://dnb.d-nb.de abrufbar.

(C) 2014 RWS Verlag Kommunikationsforum GmbH

Postfach 2701 25, 50508 Köln

E-Mail: info@rws-verlag.de, Internet: http://www.rws-verlag.de

Alle Rechte vorbehalten. Ohne ausdrückliche Genehmigung des Verlages ist es auch nicht gestattet, das Werk oder Teile daraus in irgendeiner Form (durch Fotokopie, Mikrofilm oder ein anderes Verfahren) zu vervielfältigen.

Satz und Datenverarbeitung: SEUME Publishing Services GmbH, Erfurt Druck und Verarbeitung: Druckerei C. H. Beck, Nördlingen 